Revue d'histoire de l'Amérique française

ZAS REVUE D.HISTOIRE DE L'AMÉRIQUE FRANÇAISE

\title{
Autour de la relation du P. Pierre Biard \\ Lucien Campeau
}

Volume 6, numéro 4, mars 1953

URI : https://id.erudit.org/iderudit/301551ar

DOI : https://doi.org/10.7202/301551ar

Aller au sommaire du numéro

Éditeur(s)

Institut d'histoire de l'Amérique française

ISSN

0035-2357 (imprimé)

1492-1383 (numérique)

Découvrir la revue

Citer cet article

Campeau, L. (1953). Autour de la relation du P. Pierre Biard. Revue d'histoire de l'Amérique française, 6(4), 517-535. https://doi.org/10.7202/301551ar d'utilisation que vous pouvez consulter en ligne.

https://apropos.erudit.org/fr/usagers/politique-dutilisation/ 


\section{AUTOUR DE LA RELATION DU P. PIERRE BIARD}

Le Fondateur de la Compagnie de Jésus voyait dans les échanges de lettres un puissant moyen d'édification mutuelle pour ses religieux. Il a même institué un système d'information édifiante dans les Litterae Quadrimestres, qui, de chaque maison, passant par le Provincial, aboutissaient au Général ${ }^{1}$. Admirable source de renseignements, que le Général, à son tour, déversait sur toute la Compagnie. Il le faisait sous forme de recueils manuscrits, probablement expédiés a chacun des Provinciaux, qui devaient fournir les moyens de les communiquer à leurs sujets.

Ce fut l'origine des Annuae Litterae, dont les premières sorties des presses portent la date de $1583^{2}$. Celles-ci étaient ençore réservées aux seuls Jésuites. L'activité missionnaire de la Compagnie étant d'un intérêt plus général, il importait de la faire connaître aussi au grand public. Dès la première heure, on imprima, dans ce but, les lettres de saint François Xavier ${ }^{3}$. Ces dernières rencontrèrent une grande faveur. Bientôt, de nombreuses lettres de missionnaires vont faire l'objet de semblables publications dans les principales langues européennes. Outre les histoires générales qu'elles inspirent4, ces missives servent encore à étoffer certaines compositions historiques portant sur une période récente et courte, ou un événement plus important de l'actualité missionnaire. Parfois même, les écrits de ce genre arrivent des missions tout préparés pour l'impression. On les appelle Commentarii en latin, Avvisi en italien, et souvent Rela-

1. Constitutions, VIII, I, M.

2. Auguste Carayon, Bibliographie historique de la Compagnie de Jesus ou Catalogue des ouvrages relatifs à l'histoire des Jesuites depuis leur origine jusqu'd nos jours, (Paris, 1864), 2. Le P. Carayon avertit que son relevé peut n'être pas complet.

3. La première publication est en français, imprimée à Paris en 1545. Carayon, op. cit., 62 .

4. Les premières sont d'Acosta et de Maffeo, en 1571. Carayon, op. cit., 71. 
tions $s^{5}$ dans toutes les langues autres que le français. Au seizième siècle, les Jésuites français intitulent ces documents Histoire, Advis, Discours.

Comme l'usage du terme Relation se multiplie dans les autres pays à la fin de ce siècle, il finit par apparaître en France en $1602^{6}$. Le P. Biard, en 1616, n'est que le deuxième Jésuite français à employer le vocable dans sa langue, en tête d'un ouvrage de ce genre. Le P. Le Jeune, continuant son ouvre apostolique en NouvelleFrance, l'a repris et consacré pour nommer les premières annales d'une série qui devait couvrir quarante années de missions canadiennes.

Cet article sera consacré à l'examen de la Relation du P. Biard, le premier Jésuite qui ait écrit sur les missions de la NouvelleFrance. Après une brève analyse, nous étudierons deux problèmes critiques suscités à propos de cette ouvre: le premier, posé par le désordre des numéros des chapitres; le second, celui de la chronologie, soulevé par une thèse de $M$. Gustave Lanctot.

\section{La Relation}

Le titre complet de la Relation du P. Biard se lit ainsi:

Relation/ de la/ Nouvelle/France, de ses / terres, naturel du/ pais, \& de ses habitants,/ item,/ du voyage des Pères Iesuites ausdictes/ contrées, \& de ce qu'ils y ont faict/ iusques a leur prinse par/ les Anglois/faicte/ par le P. Pierre Biard, grenoblois/ de la Compagnie de Iesius/ A Lyon,/ chez Louys Muguet,/ rue Merciere./ M.DCXVI./ Auec priuilege du Roy.

Le privilège - ou plutôt la permission du Vice-Provincial de Lyon en vertu d'un privilège royal - est daté du 23 janvier $1616^{8}$. Voici la description de l'édition originale, dont un exemplaire se

5. Le mot Relation apparait pour la première fois en italien, en 1583. Carayon, op. cit., 75.

6. Carayon, op. cit., 75.

7. Reuben Gold Thwaites, The Jesuit Relations and Allied Documents, Travels and Explorations of the Jesuit Missionaries in New France, 1610-1671, (Cleveland, 1896-1901), 3: 24. La Relation du P. Biard occupe la plus grande partie des volumes 3 et 4 de cette collection. Nous la citerons désormais en raccourci: Thw., $J R$, etc.

8. Thw., JR, 4:166. 
trouve à la Bibliothèque Municipale de Montréal. C'est un petit livre de $15 \mathrm{~cm}$. par 8 . Le titre occupe une page, et le verso est en blanc; une dédicace au Roi a trois pages, sans numéros; un avantpropos a sept pages, aussi sans numéros. Le texte couvre les pages 1 a 338. Mais il se fait un glissement de deux pages, pour tout le reste du livre, quand, après avoir atteint 264, on recommence à compter à $263^{9}$. La table compte 34 pages sans numéros, et le privilège, une page. Ce qui fait pour tout l'ensemble 387 pages.

Il ne paraît pas que l'ouvrage ait eu d'autre édition jusqu'à celle de Québec, en $1858^{10}$, où il est publié en tête des autres Relations des Jésuites. La comparaison avec l'édition originale révèle, dans le texte et jusque dans le titre, de nombreux remaniements, non seulement de l'orthographe et de la ponctuation, mais aussi $\mathrm{du}$ vocabulaire. Ces corrections sont d'ailleurs arbitraires.

Thwaites nous assure que O'Callaghan a fait à Albany, en 1871, une réimpression de cette Relation "presque en facsimile" à 25 exemplaires, vendus 25 dollars chacun ${ }^{11}$. Enfin, l'on possède la réédition critique de Thwaites, faite à Cleveland en 1897. Nous travaillerons sur celle-ci, qu'une comparaison avec l'original révèle digne de confiance.

L'intention principale du P. Biard, en écrivant cette Relation est certainement celle qu'il exprime dans l'avant-propos:

Or c'est (amy Lecteur) l'ardent desir, \& le zele de voir ceste nouvelle France, que ie dy, conquise à nostre Seigneur: qui m'a fait prendre la plume en main pour vous depeindre briefuement, \& en toute vérité ce que i'ay recogneu de ses contrées. Il y a quatre ans que i'y fus enuoyé pas (sic) mes Supérieurs: \& Dieu punissant mes pechez, i'en ay esté despuis enleué par les Anglois, ainsi que ie raconteray cy-après ${ }^{12}$.

Les titres de cette époque découragent les compilateurs de bibliographies par leur longueur, mais ils ont un avantage: ils vous

9. Thwaites ( $J R, 3: 288)$ affirme que dans l'original la page 191 est fautivement chiffrée 181. Dans l'exemplaire de la Bibliothèque Municipale de Montréal, il n'y a pas trace de faute.

10. Relations des Jesuites contenant ce qui s'est passé de plus remarquable dans les missions des Pères de la Compagnie de Jésus dans la Nouvelle-France, (Québec 1858).

11. Thw., JR, 3: 286.

12. Ibid., 3: 36 . 
donnent à l'avance la substance du volume. Si le titre ne vous intéresse pas, vous êtes dispensé de lire le reste. Celui du P. Biard, l'un des plus raisonnables, décrit, sans en omettre le plan, la matière de son livre. On y trouvera, en effet, une description exacte du pays, de son climat, de ses habitants, puis, toujours dans l'ordre indiqué, l'histoire de la mission des Jésuites jusqu'à leur retour en France. Quelques petits problèmes non prévus dans le titre ont leur solution dans les derniers chapitres.

Les descriptions sont nettes et bien bâties, et si l'auteur n'échappe pas toujours à l'habitude propre à son temps de philosopher sur divers sujets, il ne se trouve pas chez lui d'enflure ni de pompeuse rhétorique comme chez Lescarbot. Voici, par exemple, comment les Indiens se cabanent en hiver:

Quelque part qu'ils soyent arriués, la premiere chose c'est de faire du feu, \& se cabaner, ce qu'ils ont faict dans vn heure, ou deux: souuent en demy heure. Les femmes vont au bois, \& en apportent des perches, lesquelles on dispose par en bas en rond à lentour du feu: \& par en haut on les enfourche entr'elles pyramidalement, de maniere qu'elles se reposent l'vne contre l'autre droit au dessus du feu; car là est la chominée. Sur les perches on iette des peaux, ou bien des nattes, ou des escorces. Au pied des perches dessous les peaux se mettent les sacs. Toute la place à l'entour du feu est ionchée de fueilles de pin, a fin de ne sentir l'humidité de la terre: dessus les fueilles de sapin ils iettent souuent des nattes ou des peaux de loup marin aussi délicates que le velours; la dessus ils s'estendent a l'entour du feu ayant la teste sur leurs sacs. Et ce qu'on ne croiroit pas, ils sont tres-chaudement leans (sic) dedans à petit fou, voire aux plus grandes rigueurs de l'Hiuer ${ }^{13}$.

A beau mentir qui vient de loin, dit-on. Le P. Biard ne veut pas tromper, et si l'on conserve des illusions sur la Nouvelle-France après l'avoiı lu, il n'en sera pas responsable:

Ie dy cecy parce que ceste prudence importe de beaucoup a ceux qui vont defricher nouuelles contrées, ainsi que nous autres François y allons volontiers à yeux clos, \& teste baissée: croyants par exemple, qu'estants en Canada, \& ayants faim nous ne ferons qu'aller en vn'Isle, \& là escrimants d'vn gros baston à dextre, \& à senestre, autant de coups, autant

13. Ibid., 3: 76 . 
arresterons nous d'oiseaux, desquels chacun vaudra bien vn canart. Voila qui est bien dit, \& ainsi l'ont fait nos gens plus que d'vne fois, \& plus qu'en vn lieu. Cela va fort bien, si vous n'auiés iamais faim sinon au temps que ces oiseaux se trouuent en ces Isles, \& si lors mesme vous estiez proches d'eux. Car si vous en estes à cinquante, ou soixante lieues, que ferezvous ? $^{14}$

Le missionnaire est encore fin observateur des mœurs. Ayant décrit la société souriquoise comme un ensemble de clientèles gravitant autour des Sagamos, il note:

Quant quelqu'vn par exemple commence à s'émanciper, $\&$ faire le Sagamo, quand il ne rend point le tribut, quand ses gents le quittent ou que d'autres les luy soustraient; \& comm'entre nous, aussi entr'eux y a des reproches \& mespris, cestuy-la n'est quvn demy Sagamo, c'es vn nouuellement esclos, comm'vn poussin de trois iours, la creste ne luy faict que de naistre: c'est vn Sagamochin, c'est a dire, vn Aubereau de Sagamo, vn petit nain. Et à celle fin que sçachiés que l'ambition a son regne encores dessous le chaume, \& les roseapx, aussi bien que dessous les toicts dorés: Et qu'il ne faut point nous tirer beaucoup l'aureille pour apprendre ces leçons. ${ }^{15}$

Comme d'autres de ses collègues, l'ancien professeur de théologie ne dédaigne pas le calembour. Il raconte une échauffourée à laquelle il a pris une part bien involontaire. Biencourt, le fils du seigneur de Port-Royal, s'est permis d'occuper par la force le campement d'un traiteur, le capitaine Merveille, en l'absence de ceiui-ci. $\mathrm{Au}$ retour du marin, on lui lance le qui-vive, mais il répond en plaisantant. La sentinelle de Biencourt, qui n'entend pas à rire, décharge sur lui son mousquet. "Et bien merueille fut, ajoute le P. Biard, que Merueille ne fut ny tué ny blesséc."

Dans la partie historique, le narrateur poursuit un but secondaire de son œuvre, celui de réfuter les accusations qu'a lancées contre lui l'auteur anonyme d'un libelle chargé de calomnies ${ }^{17}$. Ce

14. Ibid., 3: 66 .

15. Ibid., 3: 88 .

16. Ibid., 3: 212.

17. Gabriel Marcel, Factum du procès entre Jean de Biencourt, sr de Poutrincourt et les Pères Biard et Masse, jesuites, (Paris, 1887). Ce libelle lui-même a pour titre: Factum du procez entre messire Iean de Biencourt, cheualier sieur de Poutrincourt, Baron de S. I ust, appelant d'vne part, et Pierre Biard, Euemond Massé \& consorts, soy disans prestres de la Societe de Iesus, intimez, (s.l., M.DC.XIII). 
pamphlétaire est un ami du sieur de Poutrincourt, et il ne serait peut-être pas mal aisé de l'identifier sous le nom de Lescarbot. Ce diffamateur, \& factieux, écrit le P. Biard,

commençant dés l'embarquement des Iesuites, les poursuit, comme à la trace en Canada par boys, \& rivieres, \& terres, de iour \& de nuict, en tous leurs voyages \& demeures, espiant par tout de tirer sur eux a couvert \& proditoirement quelques impostures, \& calomnies ${ }^{18}$.

A vrai dire, l'auteur de la Relation ne jouera que très modérément la note apologétique. Car, à travers tout son récit, il suit à peu près la ligne de conduite qu'il trace après avoir raconté la mort du Sagamo, Membertou:

Tel est le recit veritable de la maladie, \& mort de Membertou. sur lequel ie ne m'amuseray pas plus long temps à refuter les calomnies du factieux; estant assez \& icy, \& par tout conuaincu. Parquoy ie raconte la verite du faict sans perdre le temps à combattre les larues's.

Lescarbot et le Factum se trouvent souvent en contradiction avec le P. Biard. L'autorité de celui-ci en est-elle amoindrie? Nous ne le croyons pas. L'excès évident des attaques dirigées contre le missionnaire n'obtient qu'un résultat, celui de discréditer l'honnêteté des Biencourt, père et fils ${ }^{20}$. Leur rapporteur fidèle, Lescarbot, a quelque

18. Thw., JR, 3: 156.

19. Ibid., 3: 206.

20. Ces deux hommes, qui ne cessent d'invoquer leur qualité de gentilshommes pour obtenir créance sur parole, avaient une singulière conception de l'honneur. On sait que le père périt dans une mutinerie dont il était l'instigateur, et que nous raconterons. Le fils offrit aux Anglais de leur vendre ses services, avec toutes les fourrures de l'Acadie (Samuel Purchas, Hakluytus Posthumus or Purchas his Pilgrimes..., 20 vols., (Glasgow, 1905-1906), 19: 215). Ce témoignage de Purchas, publié d'abord en 1620, est d'autant plus précieux qu'il origine d'une source étrangère à la querelle Biard-Biencourt. Furchas, qui a connu les écrits de Lescarbot et reproduit certaines de ses accusations contre le Jésuite, a aussi puisé à une source anglaise si bien renseignée qu'elle nous paraît être Argall lui-même. Cette tentative de trahison explique suffisamment l'acharnement du jeune Biencourt contre le P. Biard, dont il s'agissait de discréditer a l'avance le témoignage compromettant. A la vérité, la manœuvre était inutile. Même attaqué et vilipendé, Biard n'a rien écrit qui pât entacher l'honneur du fils Biencourt. Au contraire, si lui-même et son père ont été jusqu'ici en si bonne posture devant l'histoire, c'est au P. Biard, aussi bien qu'à Lescarbot, qu'ils le doivent. 
honte à paraître partager leur fureur, et il se couvre, soit de l'anonymat, soit d'un faux visage d'impartialité. Adrien Huguet, le biographe de Poutrincourt, malgré l'excessive sympathie qu'il témoigne à son héros, n'ose pas prendre à son compte tout ce que ce dernier a colporté sur les Jésuites ${ }^{21}$. L'erreur serait trop grossière. Champlain, un authentique homme d'honneur, a réservé aux procédés du fils Biencourt l'une de ses rares indignations:

Est-il possible, écrit-il vers 1630, que la nation Françoise produise de tels monstres d'hommes détestables, semeurs de faussetez calomnieuses, pour faire perdre la vie à ces bons Pères ${ }^{22}$.

Nous ne pensons donc pas l'autorité du P. Biard sérieusement entamée par les assauts des Biencourt. Nous nous proposons cependant d'étudier un jour l'une ou l'autre des plus sérieuses accusations portées contre le Jésuite. Pour le moment, nous passons à la seconde partie du plan que nous nous sommes proposé, l'examen du désordre introduit dans la numération des chapitres de la Relation.

\section{Les numéros des chapitres}

Dans l'édition Thwaites, qui a reproduit les particularités de l'original ${ }^{23}$, on observe, en tête des chapitres, une grande irrégularité dans la suite des numéros. Les chapitres XI, XIX, XXII et XXIII manquent. Par contre, on a deux chapitres XXI et trois chapitres XXXII. En sorte que, de XII à XXXVIII, il ne se trouve qu'un chapitre, le dernier de ceux qui ont le numéro XXXII, qui soit

21. Adrien Huguet, Jean de Poutrincourt, fondateur du Port-Royal en Acadie, vice-roi du Canada, 1557-1615, Campagnes, voyages et aventures d'un colonisateur sous Henri IV, (Paris, 1932). Ce livre est une mine d'informations et de références; c'est dire son utilité. Toutefois, mal composé, il manque aussi totalement de sens critique. Pis encore, l'auteur ne sait pas lire les documents!

22. Laverdière, éd. Oeuvres de Champlain, (Québec, 1870), 2:777-778. Champlain savait-il que le "François meschant \& desnature" qu'il flagelle de la sorte était le propre fils de Poutrincourt? Il ne parait pas. Mais cette identification ne fait aucun doute. Elle est attestée à la fois par Lescarbot (Biggar, ed., The History of New France, 3 vols., The Publications of the Champlain Society, (Toronto, 1907, 1911, 1914), 3: 340), et par Purchas (op. cit., 19: 215-216).

23. Nous avons pu nous en rendre compte a l'examen. 
correctement nombré, les autres portant une erreur d'une, de deux, et même de trois unités.

Il est facile d'attribuer à une distraction du typographe le numéro XXI placé entre les chapitres XVIII et XX: un chiffre a pu sauter hors de sa place et XIX serait devenu XXI. Ainsi, la série serait continue depuis XII jusqu'à XXI, puis de XXIII jusqu'à $\mathrm{XXX}$. Comment expliquer l'ordre, ou le désordre, des chapitres suivants: XXX, XXXII, XXXI, XXXII, XXXII, XXXIV? Nous ne réussissons même pas à proposer une hypothèse. La seule remarque que l'on puisse faire, c'est que l'écart entre le numéro inscrit et l'ordre numérique réel, de deux unités avant XXX, n'est plus que d'une après XXXIV. Si l'un ou l'autre de ces chapitres a été inséré après l'achèvement de l'œuvre, il nous est impossible de dire lequel, tellement tous semblent nécessaires.

Reste à expliquer l'absence des numéros XI et XXII qui, les premiers, jettent le trouble dans la série. Ici, au moins, peut-on donner une raison: ces deux chapitres ont été retranchés.

Pour le chapitre XI, cela saute aux yeux. A la fin du chapitre $X$, en effet, l'auteur annonce le suivant:

Ores, puis que nous auons fait mention du Factum, escrit contre lesdits Iesuites, \& qu'il nous faudra d'ores-en-auant coup sur coup, en conuaincre les mensonges: c'est ioy a nous d'exposer, quel est ce Factum, qui son Escriuain, \& quelles causes il se dit auoir eu de sortir au monde ${ }^{21}$.

Or l'édition originale ne possède pas cette exposition. Et le chapitre XII, qui suit immédiatement dans l'état présent du texte, commence à démolir le Factum par une narration véridique des faits. Le P. Biard avait donc composé un chapitre XI, qui devait décrire le libelle et démasquer son auteur, mais ce chapitre n'a pas été publié.

La suppression du chapitre XXII est moins apparente, mais non moins certaine. A ne lire que la Relation, il reste une impression de mystère. On y fait allusion à des difficultés très graves, élevées entre Biencourt, gouverneur intérimaire de Port-Royal, et les Jésuites, difficultés qui ont "dissipé, \& rauagé tous les fruicts, \& les espé-

24. Thw., JR, 3: 242. 
rances de ce premier essartement" (ch. XXI) ${ }^{25}$, et qui ont nécessité une "réconciliation" (ch. XXIII) ${ }^{26}$ entre les deux pouvoirs. Cependant, rien de ce qui nous est raconté n'a les proportions d'une aussi considérable querelle. De plus, alors que le chapitre XXI raconte les événements de février-mars 1712 , le chapitre XXIII, qui le suit immédiatement, fait déjà l'histoire de ce qui se passe au cœur de l'été de la même année. La lecture des documents parallèles ${ }^{27}$ nous apprend que le $\mathrm{P}$. Biard omet quatre mois d'histoire, qui arriveraient entre nos deux chapitres. Et ces quatre mois ont vu la plus terrible crise qui ait secoué la petite colonie; au point que le P. Biard, supérieur de la mission, a suspendu pendant presque tout ce temps l'exercice public du culte!

Lescarbot n'a pas manqué de souligner cette omission, qui pouvait témoigner d'un certain embarras chez le Jésuite:

Le même pere Biart, écrit-il, passe souz silence sept mois de temps, sçavoir depuis Ianvier jusques à la fin d'Aoust, durant lequels y eut un divorce entre eux fort memorable, \& qui sert à l'histoire ${ }^{28}$.

Les sept mois sont un peu étirés. En réalité, les incidents omis ont occupé l'intervalle entre le 13 mars et le 25 juin 1612 .

Le chapitre XXII a donc aussi été supprimé, comme le XIième. A quel moment et pour quelles raisons, c'est ce qui nous reste à voir. Le P. Biard, rentré en France le 24 avril $1614^{29}$, a rédigé sa Relation, problablement à Amiens ${ }^{30}$, puis à Lyon, dans le courant de la même année et pendant l'année suivante ${ }^{31}$. Le manuscrit n'a

25. Ibid., 3: 242.

26. Ibid., 3: 244.

27. Lescarbot, Histoire de la Nouvelle-France, cf. Biggar, ed., op. cit., 3: 334335; Factum, 42-63.

28. Biggar, op. cit., 3: 334.

29. L'ambassadeur Spifame a Puysieulx, Londres, 3 mai 1614. Bibl. Nat., Ms. fr., vol. 15987, f. 234.

30. A la fin de mai 1614, le P. Biard écrit au P. Général et sa lettre part d'Amiens (Thw., $J R, 3: 18$ ). Dans la Relation, il mentionne la Picardie comme la province ou il vit. Voir aussi Rochemonteix. Les Jesuites et la Nouvelle-France au XVIIe siècle, (Paris, 1895), 1: 83, note 2.

31. La plus grande partie de la Relation a été rédigée en 1614 (cf. Thw., JR, 4:100); l'avant-propos parait être de 1615 , et le premier chapitre a été au moins re-

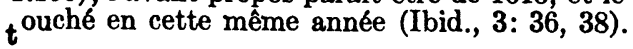


pas manqué d'être soumis à l'examen des censeurs et à l'approbation des Supérieurs. Toutes ces démarches, avec les retouches toujours nécessaires, ont occupé le reste de l'année 1615. La permission d'imprimer est du 23 janvier 1616, donnée par le Vice-Provincial de Lyon, où se trouvait l'imprimeur. Pendant que la Relation s'imprimait ainsi à Lyon, le P. Biard demeurait à Pontoise, non loin de Paris.

Un fait s'oppose à ce qu'on attribue à une décision des Supérieurs la suppression des deux chapitres en cause: c'est l'état de mutilation qui en résulte pour le livre du P. Biard. Les Supérieurs ne pouvaient guère s'objecter à une réfutation des calomnies du Factum; il est plus probable qu'ils y ont encouragé l'auteur. Et celui-ci était homme d'assez de jugement et de pondération pour garder la mesure en ces chapitres, où il ne pouvait quand même manquer de dévoiler un peu l'odieux de la conduite des Biencourt. $\mathrm{Si}$ les Supérieurs avaient trouvé la riposte trop vive, un recours leur était laissé, qu'ils se devaient d'utiliser: celui d'imposer au P. Biard une correction de son manuscrit. Le fait que les deux chapitres ont été brusquement retranchés, sans qu'on ait essayé de raccommoder le reste - ne fat-ce qu'en changeant la numérotation des chapitres - et au risque de compromettre toute la portée apologétique de l'œuvre, ce fait, dis-je, exclut l'intervention des Supérieurs et suppose que la suppression s'est opérée subitement et d'autre façon.

Ces deux chapitres formaient la pièce maîtresse de la défense du P. Biard contre les Biencourt: il leur était impossible, malgré toute la charité de leur auteur, de ne pas jeter quelque ombre sur la réputation des deux hommes. En effet, le Factum et les incidents de mars à juin 1612 révèlent leurs plus mauvais côtés. La disparition de ces chapitres, par conséquent, ne doit-elle pas être reliée à un événement qui se produisit peu avant l'impression du volume: la mort ignominieuse de Poutrincourt, arrivée le 5 décembre 1615?

H.P. Biggar, rééditant l'Histoire de la Nouvelle-France, a révélé les circonstances de cette fin tragique ${ }^{32}$. Elles s'accordent bien peu avec la laconique affirmation de Lescarbot, qui donne à son ami le

32. Biggar, ed., op. cit., 3: 532-537. 
bénéfice d'une fin glorieuse ${ }^{33}$. Il est nécessaire de résumer ici les rapports de témoins oculaires, indépendants les uns des autres, mais concordants pour la substance des faits.

C'était au cours des troubles suscités par le mariage de Louis XIII avec une princesse espagnole, Anne d'Autriche, et celui de la princesse royale Elizabeth avec le prince Philippe d'Espagne. Condé, à la tête des Princes, s'était emparé de plusieurs places fortes. Méry, petite ville que Poutrincourt avait gouvernée sous la Ligue et qui se trouvait près de sa baronnie de Saint-Just, était au pouvoir des rebelles. Le marquis de La Vieuville, lieutenant du Roi, entreprit de la reprendre, avec le concours des habitants de Troyes et de quelques seigneurs du pays, parmi lesquels Poutrincourt. Celui-ci s'était mis dans la tête de faire son profit de cette conquête et de s'assurer de nouveau le gouvernement de la ville. Méry était divisée en deux parties par un bras de rivière guéable: d'une part se trouvait la basse-ville, qui n'était pas défendue; de l'autre, la hauteville, fortifiée seulement d'une barricade de tonneaux sur la rivière, mais fermée d'une mauvaise muraille pour le reste.

Le vendredi, 4 décembre 1615, Poutrincourt et les seigneurs, ses alliés, se trouvaient installés dans la basse-ville, prise sans résistance, avec deux canons prêtés par le Lieutenant du Roi. Celui-ci avait fixé son camp sur le côté opposé de la ville, en face de la muraille. Il fit sommer le commandant de Lamets de se rendre, en tirant quelques boulets sur les murs. Les assiégés, ne pouvant résister, acceptèrent aussitôt de capituler, à condition de sortir en armes de la ville, avec les honneurs de la guerre. Poutrincourt et ses amis, à l'arrière, ignoraient ce qui se passait. De Lamets obtint du Marquis

33. Ibid., 3: 343. Nous avons cherché ce qu'avaient à dire les amis de Poutrincourt pour sa défense. Ce ne sont qu'affirmations gratuites, sans la moindre preuve, dans une chose si importante. Huguet invoque le têmoignage de Lescarbot, qui consiste en une simple affirmation. Il y ajoute celui de Jean de Laët (Noons Orbis seu Descriptionis Indiae Occidentalis Libri XVIII, Anvers, 1633, p. 60), qui a suivi Lescarbot et est encore plus laconique. Voila tout ce qu'on peut apporter pour nier la valeur de récits détaillés, écrits sur les lieux, le jour même ou le lendemain de l'événement, par des témoins. Huguet lui-même raconte l'incident sur la foi de ces récits, en leur faisant dire juste le contraire de ce qu'ils disent. Ces narrations sont au nombre de trois. Les deux premières ont été publiées par Biggar. La troisième est celle d'un manuscrit de l'avocat Waignart, conservé à Abbeville, imprimé dans le Mercure François (t. 4, année 1615: 381-383) et publié en photostat dans le volume de Huguet (face a la page 444). 
la permission d'aller les avertir, en traversant la rivière sur un bac. A cette nouvelle, Poutrincourt, qui voyait son titre de gouverneur lui échapper, n'ayant pas eu de part à la conquête, entra en fureur et fit jouer le canon contre la haute-ville, déjà au pouvoir du Roi. La Vieuville dut venir en hâte faire cesser cette mitraille scandaleuse et commander aux occupants de la basse-ville plus de tranquillité.

La nuit suivante, Poutrincourt tient conseil avec ses amis et les décide à s'emparer de la haute-ville, le samedi matin, 5 décembre, avant l'entrée des troupes royales et la sortie des vaincus. En effet, déjà les troupes du sieur de Lamets sont rangées avec leurs bagages, pour recevoir le vainqueur, quand Poutrincourt, traversant la rivière à gué avec une poignée de ses hommes, entre dans la ville. Ses alliés, faisant un détour, se sont emparés d'une porte, par laquelle ils s'introduisent aussi dans la place, avec les canons. Au même moment, par une autre porte, entrent les troupes royales et les contingents troyens. Poutrincourt, à pied, couvert d'une rondache, l'épée nue à la main gauche, le pistolet à la droite, se précipite à leur rencontre en criant: “Tue, tue. Vive Poutrincourt!" Les arquebusades ont déjà tué un cheval et blessé un soldat du Marquis. Le sieur de la Halle commande au mutin, au nom du Marquis, de cesser le feu; rien n'y fait. Le Lieutenant du Roi apparaît lui-même et prend le commandement de ses soldats. Pendant la bagarre, les vaincus de la veille, rangés en bon ordre et immobiles, assistent au combat que se livrent leurs ennemis. Les alliés de Poutrincourt, figés aussi dans un autre coin, ont bien garde d'intervenir dans une aussi folle aventure. Poutrincourt est frappé à la poitrine et tombe mort sur la place. Un bon nombre de ses hommes sont tués; son fils cadet est fait prisonnier. Le Marquis poursuivit son avantage de façon barbare en massacrant ceux qu'il put reconnaître comme partisans de Poutrincourt. Le moyen de les identifier était de tâter leurs chausses, mouillées au passage de la rivière. Les troupes alliées du seigneur de Saint-Just, peureusement arrêtées près de la porte où elles étaient entrées, n'échappèrent au carnage qu'en niant toute participation à l'entreprise.

Ainsi finit misérablement Poutrincourt, cet homme courageux et tenace, que ses incroyables malheurs rendraient sympathique s'ils n'avaient, la plupart, trouvé leur source dans son esprit faux et défiant. On comprend la pitié qui saisit le P. Biard, à la nouvelle de 
cette mort: la Relation témoigne qu'il admirait cet homme, malgré ses défauts, à cause surtout de sa bravoure et de son énergie. Il a préféré laisser sa propre réputation sans défense adéquate, plutôt que de charger davantage la mémoire d'un homme que toute la France, à ce moment, considérait avec stupeur et scandale ${ }^{34}$. C'est pourquoi il aura commandé lui-même la suppression des parties de son livre les plus compromettantes pour le défunt. Le texte souffrait de cette mutilation, mais le $\mathrm{P}$. Biard se trouvait alors trop éloigné de Lyon pour retoucher son manuscrit.

\section{La Chronologie du P. Biard et le premier voyage de Cartier}

Dignes de remarque aussi sont les distractions du P. Biard sous le rapport de la chronologie. Sont-elles le résultat de la fatigue et des misères endurées pendant de longs mois de captivité sur un navire à la dérive, au milieu d'inquiétudes et de dangers perpétuels? Ou encore viennent-elles de ce que le P. Biard n'a pas en main toutes ses notes et tous ses écrits antérieurs? Peut-être ces deux causes ont-elles joué à la fois, avec la difficulté bien connue des historiens de retenir avec précision un grand nombre de dates dans sa mémoire. La Relation, en tout cas, contient plusieurs notations chronologiques erronées, même pour des événements auxquels son auteur a été intimement mêlé. Nous possédons, heureusement, les témoignages rédigés au moment des faits, qui nous permettent de contrôler et de vérifier.

Le P. Biard avait déjà eu des distractions en chronologie. Ne l'a-t-on pas vu écrire, sur une lettre de 1612 , le millésime de 1611 ? $^{35}$ Mais, à mesure qu'il s'éloigne des événements, les erreurs se multiplient. Ainsi, la date du rendez-vous à Dieppe, donné par Biencourt pour le 27 octobre $1610^{36}$, se trouve transformée, en 1614, en celle

34. La mutinerie de Poutrincourt fit beaucoup de bruit. Dès le mois de décembre 1615, deux des récits qui nous servent de sources étaient déjà imprimés, à Paris.

35. Thw., JR, 2:104. Lettre adressée de Port-Royal au P. Général de la Compagnie de Jésus à Rome.

36. Cette date est bien certaine, donnée par les documents les plus rapprochés de l'événement: lettre du 21 janvier 1711, Thw., $J R, 1: 130$; lettre du 10 juin 1611, ibid., 1: 140 . 
du 24 octobre ${ }^{37}$. Cette même année 1614, il donne encore comme date de son arrivée à Port-Royal le 25 juin 1611, au lieu du 22 mai, jour de la Pentecôte ${ }^{38}$. Dans la Relation, il se trompe avec une sorte d'obstination. Le rendez-vous à Dieppe (27 octobre 1610) est encore à la date du 24 octobre $^{30}$. Il assigne au mois de juillet 1611 , plutôt qu'au mois de juin, le départ de Poutrincourt pour la France ${ }^{40}$, et cette fois, il affirme être arrivé à Port-Royal le 22 juin (1611), sans remarquer que la Pentecôte ne peut jamais tomber aussi tard ${ }^{41}$.

Nous ne pouvons donc, avec la même confiance que $M$. Gustave Lanctot, affirmer, sur la foi du P. Biard, que Jacques Cartier a accompagné Verrazzano en 1523-1524. C'était là une hypothèse favorite du brillant historien. Certaines convergences, pensait-il, l'incitaient à la poser. Il a enfin cru trouver un témoignage autorisé et explicite dans le P. Biard, bien que les Jésuites soient d'ordinaire, à ses yeux, sujets à suspicion ${ }^{42}$. Nous n'en voulons pas à l'hypothèse de $M$. Lanctot, qui est vraisemblable sans être exclusive, mais nous voulons lui enlever sa dernière illusion sur la créance qu'on peut accorder à un Jésuite. Le témoignage du P. Biard n'établit en aucune façon sa thèse à propos du voyage de 1524 .

Nous ignorons si M. Lanctot garde encore la conviction qui le possédait en 1947. Dans un livre de $1951^{43}$, l'abondant écrivain s'est

37. La présente erreur et la suivante se trouvent dans une relation latine, publice dans Thwaites sous le titre de "Relatio rerum gestarum in Novo-Francica missione": 2: 212: "ad octauum calendas Nouembris."

38. Thw., JR, 2: 218: "...in ante diem septimum calendas Quintiles, eumdemque Pentecostes sacrum felicissimo plane omine incidit." La contradiction est flagrante, la Pentecôte ne pouvant jamais tomber le 25 juin.

39. Thw., JR, 3:166 et 170 .

40. Thw., JR, 3:192. Le P. Biard a écrit, dans une lettre a la date du 11 juin 1611: "Le vaisseau s'est déjà éloigné. Je vais être obligé d'aller le rejoindre en canot, pour qu'il ne parte pas sans mes lettres." (Thw., 1: 190).

41. Thw., JR, 3: 180. Il faut dire, à la décharge du P. Biard que le fait d'avoir reculé son arrivée à Port-Royal de tout un mois, du mois de mai au mois de juin, a été la source d'un bon nombre de ses erreurs.

42. Gustave Lanctot, Jacques Cartier devant l'histoire. (Montréal, 1947), 128. Qu'on essaie de lire, en gardant son sérieux, la phrase suivante: "Mais, dans le texte de la Relation de 1614, comme il s'agit de faits historiques qui ne touchent ni à la religion ni à la Compagnie de Jésus, il n'entre en ligne aucune raison pour le rédacteur de ne pas adhérer à la plus stricte exactitude."

43. Gustave Lanctot, Réalisations françaises de Cartier à Montcalm, (Montréal, 1951), 15-16. On ne voit plus bien si l'auteur garde un doute sur le sujet du premier voyage de Cartier. Il affirme d'abord: "Le fait semble, d'ailleurs, nettement établ 
moins avancé, sans qu'on sache s'il a renoncé à sa première certitude. Puisqu'il ne l'a pas, que nous sachions, formellement abandonnée, il convient d'étudier ici l'autorité du P. Biard en rapport avec le point particulier qui nous occupe. Voici, pour l'essentiel, le texte sur lequel s'appuie l'historien pour tirer une conclusion définitive:

Depuis l'an 1523. Iean Verazan courut toute la coste, dés la. Floride iusques au Cap Breton, \& en prit possession au nom de François I. son maistre... De ces deux mots de Norambegue \& de Acadie, il n'en reste plus aucune memoire sur le pays; ouy bien de Canada, laquelle fut principalement descouuerte par Iaoques Cartier, l'an 1524. \& puis par vn second voyage dix ans apres, l'an $1534^{44}$.

Afin qu'on ne nous accuse pas d'exagérer l'importance accordée à ce témoignage du Jésuite, écoutons $M$. Lanctot lui-même:

Avec ce témoignage du Père Biard, d'une authenticite certaine et d'une valeur indiscutable, l'historien se trouve en présence de la pièce documentaire qui établit avec certitude la date du voyage de Cartier aux côtes canadiennes ${ }^{45}$.

L'autorite du P. Biard, sur ce point particulier, ne saurait, parait-il, soulever aucun doute. En effet, dit-on, il a dû se renseigner à Dieppe, où il fut forcé de "séjourner plusieurs mois..., par suite du refus des armateurs huguenots d'embarquer des Jésuites pour l'Acadie" ". Puis, "au retour de sa captivité en Angleterre, il dut encore, avant de rédiger son texte, consulter les auteurs et les autorités du temps"'47. Tous ces devoirs, il resterait à savoir de quelle façon le P. Biard a pu s'en acquitter. Les "plusieurs mois à Dieppe" se réduisent à quelques semaines au plus, parce que, devant le refus obstiné des Huguenots, le P. Biard s'est retiré au collège d'Eu avec

par deux documents, qui affirment sa présence en Amérique a cette date." Mais il ne produit pas ces documents, dont le plus important et le seul qui soit définitif est le témoignage du P. Biard. Et il laisse paraftre plus loin quelque hésitation: "En faveur de cette hypothese que Cartier accompagnait Verrazzano en 1524 et en 1528, 'établit encore toute une convergence, sinon de témoignages, du moins, de probabilités, aussi suggestives que lumineuses."

44. Thw., JR, 3: 40.

45. G. Lanctot, Jacques Cartier devant l'histoire, 130.

46. Ibid., 129.

47. Ibid., 129. 
son compagnon ${ }^{48}$. D'ailleurs, durant leur séjour à Dieppe, les Jésuites avaient bien d'autres occupations que la recherche historique. Quant à une enquête approfondie, au moment de la rédaction, le missionnaire a bien pu ne pas la faire sur ce point, très secondaire a son point de vue.

Quoi qu'il en soit des possibilités, une chose est certaine: le témoignage du $\mathrm{P}$. Biard ne peut être apporté en preuve de la thèse de M. Lanctot. Car il ne dit nullement que Cartier a fait le voyage de 1524 en compagnie de Verrazzano, et il ne peut vouloir le dire. Il est facile, en effet, de montrer que, dans son esprit, $1^{\circ}$ le voyage de Verrazzano et celui de Cartier sont tout à fait distincts, et que $2^{\circ}$ le P. Biard s'est certainement trompé dans ses notations chronologiques.

M. Lanctot dépense beaucoup de subtilité et d'érudition à prouver l'identité des deux expéditions, d'après les expressions du Jésuite. On devra cependant avouer que ce dernier n'aurait pas parlé autrement qu'il n'a fait s'il avait voulu distinguer les deux voyages. Les dates, certes, ne coïncident pas, ni dans le texte cité plus haut, ni dans les autres endroits où le P. Biard aborde la question. Il en est ainsi, par exemple, dans la Relatio rerum..." “...Dix neuf ans plus tard (après 1504, i.e. en 1523), Jean Verrazzano, Florentin, puis, en vingt-quatre ainsi qu'en trente-quatre, Jacques Cartier, Français breton... ${ }^{49}$ " Puis, dans cet autre, qui paraît clore la discussion:

5. D'auantage tous confessent, que par le commandement du grand Roy François Iean Verazan print possession de cesdictes terres au nom de la France; commençant dés le 33. degré d'éleuation iusques au 45. Ce fut par deux voyages desquels le dernier fut faict l'an 1523. il y a quatre vingts $\&$ dix ans.

6. Outre plus, Iacques Cartier entra premier dans la grande riuere par deux voyages, qu'il y fut, \& descouurit les terres de Canada. Son dernier voyage fut l'an $1534^{50}$.

48. Thw., $J R$, 3: 174 .

49. Ibid., 2: 202. Le texte latin est de lecture difficile et de ponctuation défectueuse. C'est pourquoi nous devons admettre que notre traduction contient une bonne part d'interprétation, fondée à la fois sur la valeur des vocables (surtout item ac) et sur les textes parallèles. Voici le texte latin: "Vndeuicesimo post anno, Ioannes Verazanus Florentinus, vicesimo item, ac tricesimo quarto Iacobus Quarterius Gallus, Brito..."

50. Thw., $J R, 4: 104$. 
Il apparaît clairement, par les termes du P. Biard, que ce qu'il estime comme le dernier voyage de Verrazzano a eu lieu en 1523, tandis que le premier de Cartier, daté comme on sait de 1524, est tout différent.

$\mathrm{Au}$-dessus de toute controverse, à coup sûr, est la différence des trajets dans les deux expéditions. Pour le P. Biard, Verrazzano, en 1523, a parcouru la côte depuis "le 33. degré d'éleuation iusques au 47.", "toute la coste, dés la Floride iusques au Cap Breton, \& en prit possession au nom de François I. son maistre." Il n'a pénétré ni dans le golfe, ni dans le fleuve: personne, d'ailleurs ne l'a jamais prétendu. Voici maintenant ce que l'auteur de la Relation écrit de Cartier: il "entra premier dans la grande riuiere par deux voyages qu'il y fut, $\&$ descouurit les terres de Canada." Comme le P. Biard ne connaît que deux voyages de Cartier, l'un en 1524 et l'autre en 1534, il faut conclure que, dans sa pensée, le Malouin est entré dans le Saint-Laurent pour la première fois en 1524. Ce qu'il n'accorde nullement pour Verrazzano.

M. Lanctot est déjà peu excusable d'avoir ignoré les précédents témoignages, mais il ne l'est plus du tout quand lui échappe le sens du texte qu'il cite au long dans son ouvrage ${ }^{51}$. Il n'a pas vu que Canada se trouve opposé à Norambègue et Acadie: "De ces deux mots de Norambegue \& de Acadie, il n'en reste plus aucune memoire sur le pays; ouy bien de Canada, laquelle fut principalement descouuerte par Iacques Cartier, l'an 1524". Le P. Biard, qui emploie d'ordinaire le mot Canada en un sens général, le restreint ici explicitement, et $M$. Lanctot reproduit lui-même la définition qui en est donnée:

Parce que Canada (du quel nom aussi on l'appelle communement) n'est point à proprement parler toute ceste tenuë de pais qu'on nomme Nouvelle France; Ains est celle tant seulement, laquelle s'estend au long des rivages du grand Fleuve Canadas, et le golfe de S. Laurens; qui n'est seulement que la partie la plus Septentrionale de la Nouvelle France...."

Par conséquent, de ce Canada découvert par Cartier en 1524 se trouve positivement exclue la côte atlantique explorée par Verraz-

51. G. Lanctot, Jacques Cartier devant l'histoire, 123-125.

52. Nous citons d'après le texte de M. Lanctot. 
zano en 1523. Les deux voyages ne peuvent nullement être confondus en un seul.

Il est à peine nécessaire, après cela, de montrer que les dates du $P$. Biard sont inexactes. Ce premier voyage de Cartier, par lequel il a découvert le "Canada", c'est-à-dire les rives du fleuve et du golfe Saint-Laurent, tout le monde sait qu'il a eu lieu en 1534, et non en 1524. Quant au second voyage, celui que le Jésuite date de 1534 , on ne peut douter qu'il soit celui de 1535-1536, puisqu'il y rattache le premier hivernement à Québec:

De plus, sur un si vaste territoire, en de frequentes expeditions, et durant plus d'un siècle, les Français ont fondé cinq établissements. Le premier a été érigé par Jacques Cartiar dans son second voyage, non pas à Sainto-Croix d'aujourd'hui, qui est d'accès difficile et dangereux, mais à peu pròs sur les lieux où se trouve maintenant Québec....3"

Cette modeste analyse, qui se rapporte, croyons-nous, à la critique historique, ne saurait déplaire à $M$. Lanctot, passionné pour cette discipline. Est-il hors de propos de lui faire remarquer que celleci est née avant lui ? Si "parmi les auteurs, ni Charlevoix, ni Margry, ni Harrisse, ni Baxter, ni Pope, ni Biggar, ni Groulx, ni Martin, n'ont vu ce qu'ils avaient sous les yeux ${ }^{54}$ ", c'est peut-être parce qu'ils avaient assez de sens critique pour ne pas s'y laisser prendre. Avant eux, Champlain avait montré la même sagacité. Dans une pièce dont il emprunte les idées, le plan, et même d'abondants extraits textuels, à la Relation du P. Biard, il corrige, sans faire d'éclat, la date du second voyage ${ }^{55}$.

\section{CONCLUSION}

L'autorité du P. Biard ne se trouve pas substantiellement amoindrie pour tout cela. Elle est ce que sera, en général, celle de toutes

53. Thw., $J R$, 2:208. "Porro in tam immenso terrarum ambitu, frequentibus expeditionibus, annis amplius centum, Franci domicilia omnino quinque constituerunt, quorum primum posuit Iacobus Quartierus posteriore sua nauigatione, non ad Sanctae Crucis, quae nunc est, importuosas angustias, \& cautes: sed in is paene vestigiis vbi nunc est Quebecum..."

54. G. Lanctot, Jacques Cartier devant l'histoire, 123.

55. Laverdière, Éd, Oeueres de Champlain, 2: 1310. Cf. Thw., JR, 4: 106. 
les autres Relations. La haute qualité morale et intellectuelle des hommes qui les ont écrites justifient la confiance que l'on met en leurs dires. Ils ont pu se tromper parfois. Prêtres, ils n'ont pas voulu tout dire, de peur de scandaliser; quand ils ont dú écrire des choses pénibles, ils l'ont fait à contre-cœur, parce qu'ils s'y croyaient forcés. Mais ils n'ont jamais voulu tromper. Aussi peut-on les croire lorsqu'ils affirment être des témoins directs. S'ils s'appuient sur le témoignage d'autrui, leurs affirmations valent ce que valent leurs sources. Ils mettent de la conscience à s'informer, mais cette conscience doit se mesurer à l'importance du détail envisagé, par rapport au but qu'ils poursuivent.

Les missionnaires de la Nouvelle-France n'ont jamais prétendu être des historiens ou des savanst. Ils sont d'abord des prêtres, qui ont en vue le bien des âmes: de leurs néophytes à qui il est utile d'exciter les sympathies françaises; de leurs compatriotes, à qui il est bon de connaitre les manifestations d'une foi toute nouvelle. Leur ministère les engage jusqu'au cœur d'un continent neuf, leur fait ausculter les premières pulsations d'une histoire naissante, et c'est pourquoi ils sont d'incomparables témoins, tout à la fois les plus à même d'observer avec précision et les plus libres de passions et de convoitises.

Lucien Campeau, s.J.

AVIS - LA REVUE D'HISTOIRE DE L'AMERIQUE FRANÇAISE vit de ses abonnements, et rien que de ses abonnements. Elle compte que vous renouvellerez le vôtre, dès ces jours-ci, pour ne pas manquer le no de juin prochain, ler de la 7ème année. 